\title{
Erratum to: Application of Rock Mass Characterization for Determining the Mechanical Properties of Rock Mass: a Comparative Study
}

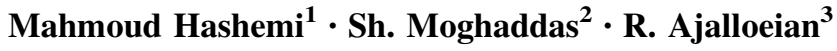

Published online: 18 April 2017

(C) Springer-Verlag Wien 2017

\section{Erratum to: Rock Mech Rock Eng (2010) 43:305-320 \\ DOI: $10.1007 / \mathrm{s00603-009-0048-y}$}

\section{Forward by the Editor in Chief (G. Barla)}

Dr. Nick Barton has recently informed the Editor that during a recent scrutiny of the above paper he found an error in Table 2, i.e. "the Q-RMR formula is $\log 10$ not $\ln$ ". Therefore, Figures 7, 8 and 14 and 15 show extremely unrealistic values for anything related to $\mathrm{Q}$. Papers published in the Journal are open for discussion up to 3 months following online publication. After this time, a correction in a paper can only be made in the form of an erratum, which will be hyperlinked to the article. The erratum prepared by Dr. Hashemi and co-authors is reported below.

The authors examined the paper thoroughly and would like to make the following statements:

The online version of the original article can be found under doi:10.1007/s00603-009-0048-y.

Mahmoud Hashemi

m.hashemi@eng.ui.ac.ir

1 Department of Civil Engineering, Faculty of Engineering, The University of Isfahan, 81744-73441 Isfahan, Iran

2 Engineering Geology, Sabir Engineering Co., Tehran, Iran

3 Department of Geology, Faculty of Science, The University of Isfahan, Isfahan, Iran
1. The authors must acknowledge the error done in applying the Q-RMR relation proposed by Barton (1995). The formula must have contained "log 10", but unfortunately, the actual calculation in applying the formula was based on using "ln". Therefore, the authors must apologize for the inconvenience caused due to the error. This has affected Table 2 and then Fig. 8, which is based on comparing the Q-RMR relations with Sabzkuh tunnel data. Corrected Table 2 and Fig. 8 (along with their captions) are presented below.

2. Corrected Fig. 8 shows that the relation by Barton (1995) overestimated the RMR values for the Sabzkuh tunnel data. Therefore, the conclusion presented in the paper is still valid as: "the closest relation to the Sabzkuh tunnel data is the one proposed by Rutledge and Preston (1978)". Please note that the relation by Kumar et al. (2004) (based on Nathpa-Jhakri project, India) is the same as the relation by Barton (1995) in corrected Fig. 8.

3. The authors must acknowledge the error done in applying the rock mass strength relation proposed by Barton (2002) and its comparison with Sabzkuh tunnel data. Therefore, the authors must apologize for the inconvenience caused due to the error. So, the error has affected Fig. 14 that (along with their captions) is presented below. 
Table 2 Comparison of various correlations among the rock mass classifications

\begin{tabular}{|c|c|c|c|}
\hline Researcher(s) & Correlation & Relation no. & Estimated parameter \\
\hline Bieniawski (1976) & $\mathrm{RMR}=9 \ln \mathrm{Q}+44$ & $(8)$ & RMR from $\mathrm{Q}$ \\
\hline Rutledge and Preston (1978) & $\mathrm{RMR}=5.9 \ln \mathrm{Q}+43$ & (9) & RMR from $\mathrm{Q}$ \\
\hline Moreno (1980) & $\mathrm{RMR}=5.4 \ln \mathrm{Q}+55.2$ & $(10)$ & RMR from $\mathrm{Q}$ \\
\hline Cameron-Clarke and Budavari (1981) & $\mathrm{RMR}=5 \ln \mathrm{Q}+60.8$ & (11) & RMR from $\mathrm{Q}$ \\
\hline Abad et al. (1984) & $\mathrm{RMR}=10.5 \ln \mathrm{Q}+41.8$ & $(12)$ & RMR from $\mathrm{Q}$ \\
\hline Kaiser and Gale (1985) & $\mathrm{RMR}=8.7 \ln \mathrm{Q}+38$ & (13) & RMR from $\mathrm{Q}$ \\
\hline Al-Harthi (1993) & $\mathrm{RMR}=9 \ln \mathrm{Q}+49$ & (14) & RMR from $\mathrm{Q}$ \\
\hline Barton (1995) & $\mathrm{RMR}=15 \log \mathrm{Q}+50$ & $(15)$ & RMR from $\mathrm{Q}$ \\
\hline Tugrul (1998) & $\mathrm{RMR}=7 \ln \mathrm{Q}+36$ & (16) & RMR from $\mathrm{Q}$ \\
\hline \multirow[t]{5}{*}{ Kumar et al. (2004) } & $\mathrm{RMR}=6.4 \ln \mathrm{Q}+49.6$ & $(17)$ & RMR from $\mathrm{Q}$ \\
\hline & $\mathrm{RMR}=5.4 \ln \mathrm{RMi}+54.4$ & $(18)$ & RMR from RMi \\
\hline & $\mathrm{RMi}=0.5 \ln \mathrm{Q}^{0.93}$ & (19) & RMi from Q \\
\hline & $\mathrm{RMi}=1.5 \ln \mathrm{Q}^{0.72}$ & (20) & \\
\hline & $\mathrm{RCR}=8 \ln \mathrm{N}+42.7$ & (21) & RCR from $\mathrm{N}$ \\
\hline
\end{tabular}

Fig. 8 Correlated data from the Sabzkuh tunnel, along with the other correlations available in the literature (ten cases)

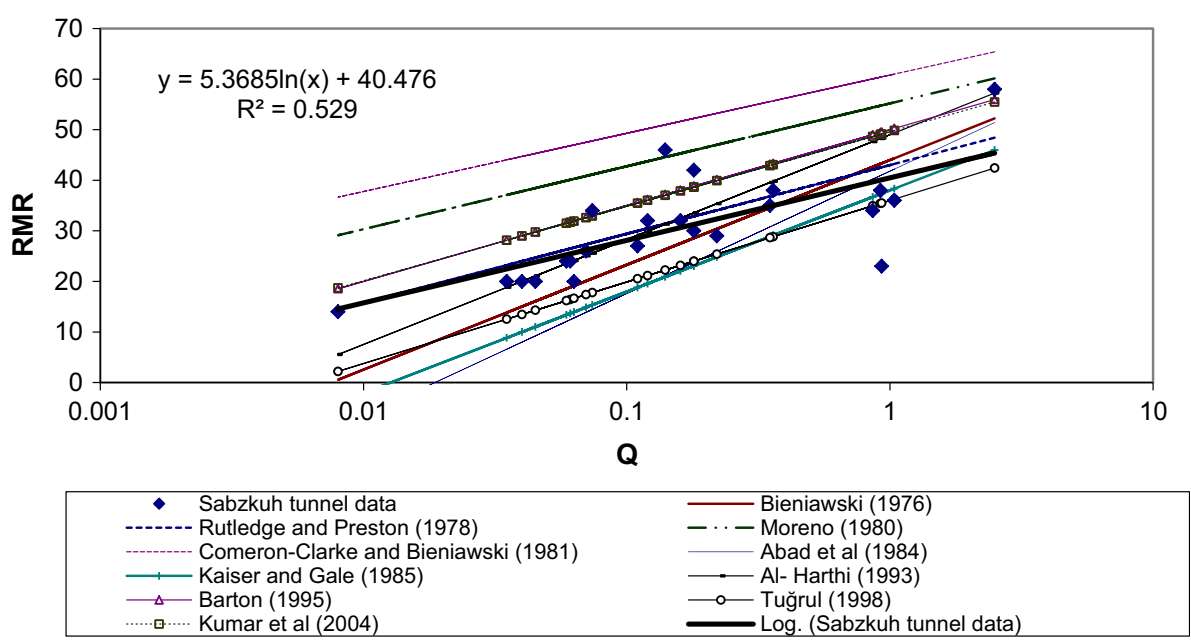

4. Corrected Fig. 14 shows that the relation by Barton (2002), unlike the conclusion provided in the paper, does not yield the low values as compared to other relations, but it provides values more than the average and close to the relation by Bhasin and Grimstaad (1996). The remaining figures, tables and conclusions are still valid and need not be corrected.

Therefore, the second paragraph of Section 4.1.2 in the paper is to be corrected as:

The relations proposed by Goel (1994) and Singh (1993) estimate high values (upper bound) for $\sigma_{\mathrm{cm}}$, whereas the relation proposed by Yudbir et al. (1983) yields low values (lower bound). The relation by Barton (2002), unlike the conclusion provided earlier in the paper, does not yield the low values as compared to other relations, but it provides values more than the average and close to relation by Bhasin and Grimstaad (1996).

Therefore, the fifth paragraph of Section 5 of the paper needs to be corrected as:

The relations proposed by Goel (1994) and Singh (1993) estimate high values (upper bound) for $\sigma_{\mathrm{cm}}$, whereas the relations proposed by Yudbir et al. (1983) yield low values (lower bound); this shows a wide range for $\sigma_{\mathrm{cm}}$. For the upper bound relations, the variation of $\sigma_{\mathrm{cm}}$ is very sensitive to variation in the input parameter. The relation by Barton (2002) provides values more than the average and close to relation by Bhasin and Grimstaad (1996). 
Fig. 14 The rock mass strength estimated using relations available in the literature

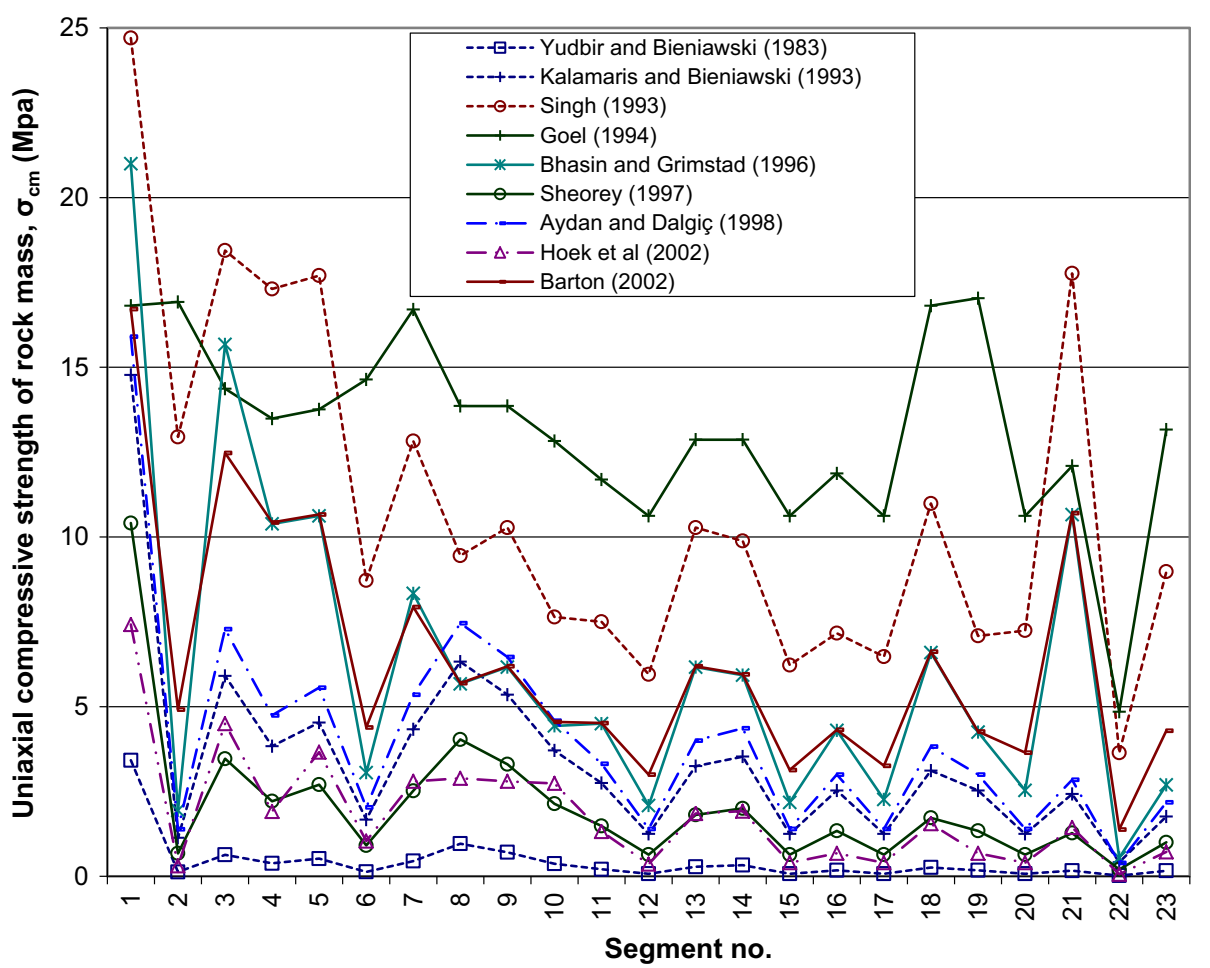

5. Out of nearly 40 relations (adopted from over 60 references) applied in the paper, there are only two errors in the RMR-Q correlation (Barton 1995) and rock mass strength (Barton 2002) relations that have affected only Figs. 8 and 14, and Table 2. These have not affected the rest of the conclusions including those reported in Figs. 1-7, 9-13, 15, and Tables 1, 5.

6. Regarding the rock mass classifications in the paper, it is noted that the rock mass classification systems are applied independently from each other. 